\title{
A Formally Verified Mutual Authentication Protocol for Low-Cost RFID Tags
}

\author{
Sarah Abughazalah, Konstantinos Markantonakis, Keith Mayes \\ Smart Card Centre-Information Security Group (SCC-ISG) \\ Royal Holloway, University of London \\ Egham, Surrey, TW20 OEX, UK
}

\begin{abstract}
In this paper, we propose a lightweight mutual authentication protocol for low-cost Radio Frequency IDentification (RFID) tags. Although RFID systems promise a fruitful future, security and privacy concerns have affected the proliferation of the RFID technology. The proposed protocol aims to protect RFID tags against a wide variety of attacks and especially Denial of Service (DoS) attacks. We found that the majority of the proposed protocols failed to resist this kind of attack. To analyse our proposed protocol, we provide an informal analysis. In addition, we formally analyse the security of the proposed protocol via using automated formal verification tools such as CasperFDR and AVISPA. We also employed an up-to-date privacy model to evaluate the privacy of the RFID protocol. The results show that the proposed protocol achieves tag's data secrecy, privacy and authentication under the presence of a passive adversary.
\end{abstract}

\section{Introduction}

RFID is a wireless technology that uses radio signals to identify tags attached to objects. It provides more advantages than a typical Automatic Identification Technologies such as bar codes; thus, it started to conquer the new mass markets [1].

It is composed of three main components, namely, a tag, a reader and a backend server. The reader broadcasts a radio frequency $(\mathrm{RF})$ signal to power, communicate and receive data from RFID tags without physical contact. The RFID tag is an identification device attached to an item; it transmits the stored information to the nearby reader(s) through the RF channel. The reader sends the tag's data to the backend server which stores data about the RFID tags it manages [2].

RFID is being used in many applications that require high level of security and privacy such as in access control systems, identification of products in the supply chain or payment system [3]. Therefore, it needs to use different security measures to protect against feasible attacks. The wireless communication between the tag and reader might allow an attacker to eavesdrop on a session, modify the transmitted messages, and prevent some messages from reaching their target. Moreover, if the tag's data is sent in the clear or fixed to any reader, a malicious reader can obtain tag's data and/or track a specific user or object location [4].

Another area that affects the adoption of RFID systems is performance. A low-cost RFID tags cannot perform computationally intensive security cryptographic functions, as it offers tightly constrained computational power and storage capacity [5]. Therefore, it supports simple bitwise operations such as XOR, and concatenation operators, and a Pseudo Random Number Generator (PRNG).

In order to ensure privacy and security of the proposed protocol, we use hash functions. Best practices should be used for the implementation of the hash functions. For example, the authors in [5] presented a hardware implementation of Keccak, which has been selected as the winner of the NIST SHA-3 competition in 2012, which aims to use the lowest power. The designed hardware can be implemented in RFID tags with only 5522 gates, and consumes $12.5 \mu \mathrm{W}$ of power.

In this paper we aim to avoid the security and privacy issues found in previous related work and in the meantime improve the performance of the RFID systems. In particular, we found that some of the proposed protocols $[6,7,8,9,10,11]$ failed to resist desynchronisation attacks.

In addition, there are other security and privacy issues that are not addressed previously such as replay attacks $[12,13]$, tag and server impersonation attacks $[14,12,8,7,6,15,16,17]$. Moreover, some recent research focused on the security side and ignored performance $[7,8,13,12,11,17,16,18]$, which require the server to search all tags in the system in order to identify a single tag.

To confirm that the proposed protocol meets the main secrecy, privacy and authentication requirements, we verify its secrecy and authentication using two model checking tools namely CasperFDR [19] and AVISPA [20]. Moreover, the privacy of the protocol is tested using a privacy model proposed by [21] to evaluate the privacy of the RFID protocols. The results of these models show that the proposed protocol achieves 
tag's data secrecy, privacy and authentication under the presence of a passive adversary.

The rest of this paper is organized as follows: in Section 2, the protocol's main goals and requirements are discussed. In Section 3, we briefly review some related work and examine their weaknesses. In Section 4, we explain our proposed protocol in detail. In Section 5, we analyse the proposed protocol with respect to informal analysis, privacy model, and mechanical formal analysis using CasperFDR and AVISPA. In Section 6, we provide the concluding remarks.

\section{Goals and requirements of the proposed protocol}

When designing an RFID protocol, consideration should be given to the following:

Privacy: We need to take into account that the tag's data should remain secret and not revealed to any malicious reader, thus providing anonymity to the tag. Another notion related to privacy is untraceability; if the data being sent from the tag to the reader is static or linked to data sent previously, the tag's holder location can be tracked without his knowledge. Therefore, the protocol should provide a mechanism to protect the tag's data from being breached.

Security: Due to the wireless communication between the tag and reader, a passive attacker can observe and manipulate the communication channel between the readers and tags. In this paper, we focus on three common techniques to violate the secrecy of the system namely replay attack, desynchronisation attack and tag and server impersonation attacks.

To elaborate, the designed protocol should resist the following attacks [22]:

- Resistance to replay attacks: The adversary can eavesdrop on the communication between reader and tag, obtain the exchanged message(s) and resend it repeatedly. Therefore, the generated messages should be fresh to the protocol session to protect against replay attacks.

- Resistance to desynchronisation attacks: The adversary can modify the flow of the messages and block messages from reaching their target causing desynchronisation between the two legitimate parties. Therefore, the main server should store the old and new values of the tag in order to authenticate the tag and reach synchronisation even if the attacker blocked any messages.
- Resistance to tag and server impersonation attacks: In this attack, the attacker sends a message to the server that claims to come from a legitimate tag, and this message fabrication enables the attacker to masquerade as a legitimate tag and vice versa. Hence, the responses should be encrypted so that creates no meaning to the attacker.

- An active attacker can physically compromise the RFID tag's memory and obtain the secret data. Active attacks are beyond the scope of this paper.

Mutual authentication: The protocol should provide a mutual entity authentication, where the communication should take place between legitimate entities such as tag, reader and server and provide assurance to the receiver (server) about the identity of the sender (tag) and vice versa.

\section{Related Work}

We start with the first privacy-enhancing RFID protocols proposed by [14]. Weis proposed a protocol [14] namely the hash-based access control (HAC) (referred here as WP). The proposed scheme uses the saved secret key $(\mathrm{K})$ and hash function $(\mathrm{H})$ to lock the tag. The tag calculates the hash of the key (HK) and sends it to all queries it receives. To unlock the tag, the server sends $(\mathrm{K})$ in the clear to the tag and the tag checks that $\mathrm{H}(\mathrm{K})$ is equal to the stored (HK); if they are equal, then the tag unlocks itself and replies with its (ID). Kim et al. [23] formally analysed this protocol by using CasperFDR and the verification demonstrates that an intruder may obtain the secret key and the tag's ID, as they are sent in the clear. Moreover, the value of (HK) is static; thus can be traced by an intruder.

An extension of the hash lock scheme based on Pseudo Random Functions (PRFs) has also been proposed in [12] (referred here as WP2). In this scheme, a tag generates a random number (R) and sends H(ID, R) and R, where ID is the tag's ID. To identify a tag, a server computes the hash of each stored ID until it finds a match with the value it receives from the tag. Then, the server sends the matching ID to the tag in clear to unlock it. This approach, however, still vulnerable to replay attacks and tag impersonation attacks as shown in [24].

Ohkubo et al. [13] suggested an alternative RFID hash chained protocol (referred to here as OP). This protocol focused on protecting the RFID privacy by achieving forward security, which is the knowledge of a tag's current internal state could help identify the tag's past 
interactions allowing tracking of the tag owner's past behaviour [24]. The tag and server store a secret (s). The tag updates (s) in every session by using a hash function (h). Then in the next transaction the tag uses a second hash function (g) and sends $\mathrm{g}\left(\mathrm{h}\left(\mathrm{s}_{\mathrm{i}+1}\right)\right.$ to the server where $\left(\mathrm{s}_{\mathrm{i}+1}\right)$ is the updated secret to be used in the next transaction. This scheme achieves forward security. However, this scheme is still vulnerable to replay attacks [25]. In [25], the author proposed an extended protocol by adding random numbers to the exchanged messages to avoid reply attacks.

Molnar et al. [26] suggested an RFID mutual authentication protocol (referred here as MP). This approach uses the challenge-response protocol as well as PRNG functions to provide privacy for RFID system. The tag and server share a key $(\mathrm{k})$ which is used in calculating the PRNG functions. The server sends a random number ( $\mathrm{r} 1)$ as a challenge to the tag. The tag sends $M 1=I D \oplus f_{k}(0\|r 1\| r 2)$ where (f) is a pseudo random number function, and a random number (r2) to the server in order to be authenticated. Once the server successfully authenticates the tag, it sends M2=ID $\oplus \mathrm{f}_{\mathrm{k}}(1\|\mathrm{r} 1\|$ r2) to be authenticated by the tag. However, this protocol does not resist traceability as the value of the secret key $(\mathrm{k})$ is fixed; hence the attacker can trace the previous communications related to this tag [24].

Dimitriou [9] proposed another RFID authentication protocol to enforce user privacy (referred here as DP). This approach uses a challenge-response protocol, hash function, and keyed hash functions. The tag only stores the tag's identifier (ID), which serves as a key to calculate the keyed hash function. The server stores the tag identifier (ID) and the hash of the tag identifier (HID), used as an index to retrieve the tag's data. When the server successfully authenticates the tag, it updates (ID) to g(ID), where $(\mathrm{g})$ is a one-way function and sends a third message to the tag. The tag checks the received value of the third message. If the check is successful, the tag updates (ID) to $g($ ID). This protocol is still vulnerable to DoS attacks [24]; if the third message (M3) sent by the server is blocked by an attacker, then the server will update the identifier (ID) while the tag keeps the old value of the identifier, resulting in a desynchronisation between the server and tag [24].

Duc et al. [8] presented an RFID mutual authentication protocol conforming to the Electronic Product code (EPC) Class 1 Generation 2 standard (EPCCIG2) (referred here as DucP). This scheme uses simple primitives such as PRNG and Cyclic Redundancy Code
(CRC) functions, as they are supported in the EPCC1G2 standard. The PRNG is used for updating the secret key while the CRC is for detecting any errors occur during the transmission of the messages. The server and tag store a secret key, which is updated when the reader sends an end session to both ends. However, this scheme still has some weaknesses such as, vulnerability to DoS attacks, tag impersonation attacks, and traceability [7].

Chien et al. [8] introduced another mutual authentication protocol for RFID conforming to the EPCC1G2 standard; this is an improved version of Duc et al. protocol (referred here as $\mathrm{CP}$ ). The proposed protocol requires the server and tag to produce random numbers to prevent replay attack. The tag keeps a static EPC as the tag identifier, and an access key $(\mathrm{K})$ and authentication key $(\mathrm{P})$, which are updated after each successful authentication. The server also maintains the same values as well as the old and new access and authentication keys to avoid DoS attacks and prevent forward traceability. The protocol uses simple cryptographic primitives, such as a PRNG and a CRC. After the server authenticates the tag, it updates the data except for the EPC identifier which is static. This protocol is vulnerable to tag impersonation attacks, and tracking of previous transactions as cited in [24]. Moreover, according to [15], this protocol is prone to DoS attacks and server impersonation attacks. Finally, [27] shows that this protocol permits location tracking due to the use of the linear properties of CRC which is used as a checksum algorithm.

Another lightweight RFID mutual authentication protocol was proposed in [10] using a Shrinking Algorithm [28] (referred here as $\mathrm{SG}$ ). In this protocol a shrinking algorithm generates a different random key that is used for the encryption purpose. The tag generates an encryption key (CSGK1) using the shrinking algorithm that takes a shared secret between the tag and the database (K1) as an input, and computes $\mathrm{M}=\mathrm{CSGK} 1 \oplus(\mathrm{ID} \| \mathrm{S})$, where ID is the tag's ID and $\mathrm{S}$ is a random number generated by the reader. Once the database authenticates the tag, it generates another random encryption key (CSGK2) using the shrinking algorithm, and computes ID $^{\prime}=\mathrm{CSGK} 2 \oplus$ (ID). Finally, both of the tag and database will update their values.

The authors claim that their protocol reaches the synchronization between the tag and database by maintaining a list of current and previous values in the database. However, we found that this protocol is vulnerable to a desynchronisation attack as if the attacker blocks the database's message from reaching the tag just twice then the tag's data will not match the server's data. Moreover, if the attacker tampered with Meta-id, 
which is used for retrieving the tag's data in the database, then the database will not be able to retrieve the tag's data and thus fails to authenticate the tag.

Song et al. [6] proposed an efficient RFID authentication protocol for low-cost tags (referred here as SP). This protocol uses the hash function, Message Authentication Code (MAC) and PRNG. Each tag stores the hash of a secret (s) namely (t), and the server stores the old and new values of the secret ( $\left.s_{\text {new }}, s_{\text {old }}\right)$, the hashed secret $\left(\mathrm{t}_{\text {new }}, \mathrm{t}_{\mathrm{old}}\right)$ and the tag's information (D). This scheme uses a challenge-response protocol and supports updating data when the mutual authentication is achieved. Cai et al. [29] presented a paper showing that Song et al.'s protocol does not provide protection against tag impersonation attacks. Moreover, Rizomiliotis et al. [30] found that an attacker can impersonate the server even without accessing the internal data of a tag and launch a DoS attacks.

A new version of the SP has been proposed in [24] (referred to here as the SP2). The protocol uses the same data as in the SP except that the construction of the exchanged message (M2 and M3) has been changed. In the new version of the protocol, the author claimed that the proposed protocol resists DoS attacks. However, we discovered that an attacker is able to desynchronise a tag without even compromising the internal data stored in the tag [31].

Poulopoulos et al. [11] proposed an RFID mutual authentication protocol using a hash function and PRNG (referred here as PP). The protocol uses a challenge response scheme. The exchanged messages are protected using a hash function. The value of the secret key $(K)$ in the tag is updated after each successful authentication. The server stores the old and new values of the identifier (ID) and (K), and they are updated after authenticating the tag. However, we found that this protocol is vulnerable to desynchronisation attack [32].

Yeh et al. [15] proposed an improved version of Chien et al.'s RFID authentication protocol conforming to the EPCC1G2 standard (referred here as YP). The data kept in the server and tag is the same as in Chien et al.'s protocol, except that their protocol uses an index (C) to avoid DoS attacks and database overloading. The initialisation and authentication phases are quite different from those of Chien et al.'s protocol; they do not use CRC functions; only PNRG functions, thus blocking the bad linear properties of the CRC function [27]. Although this protocol prevents DoS attacks, it is still vulnerable to forward traceability, tag impersonation attacks, and server impersonation attacks as pointed in [16].
Another RFID mutual authentication protocol called O-FRAP was proposed in [18]. In this protocol, each tag and the server share a key $(\mathrm{K})$ and a pseudonym ( $\mathrm{r}$ ) which are updated in every session. In this paper, the authors claimed that the proposed protocol resists DoS attacks and achieves forward security. However, [33] stated that this protocol is vulnerable to a traceability attacks and it fails to provide forward security by corrupting the target tag at the point after the tag outputs (Accept).

An improved version of Yeh et al.'s protocol is proposed in [16] (referred to here as YoonP). Their protocol uses the same data as Yeh et al.'s protocol and the initialisation process is also identical, but the authentication phase is quite different by adding a secret session random number (r2) to the exchanged massage (M1). Yoon et al. claims that the proposed protocol provides more security than that of Yeh et al.; however, [34] showed that eavesdropping on only one session of the protocol can reveal the tag's secret data, as the length of data generated from PRNG is only 16 bit strings, which makes it easier for the attacker to do an exhaustive search to find the pre-image of the stored data; it is thus easy to launch a traceability attacks, DoS attacks, tag impersonation attacks, and server impersonation attacks.

In [17] the authors proposed an RFID mutual authentication protocol which is an improved version to the protocol proposed in [13] (referred here as HP). Their protocol supports the updating of the server's and the tag's secret data as well as using different hash functions for the authentication. In this scheme, the whole protocol depends on the secrecy of only one secret value $\left(\mathrm{sk}_{\mathrm{ID}, \mathrm{i}}\right)$ which if it is compromised the protocol will be affected. Also, the value of the ID is static, hence it can be tracked. Moreover, we found that this protocol is still vulnerable to server impersonation attacks and desynchronisation attacks (only if the secret value is compromised).

Hence, a new RFID mutual authentication protocol that can resist such attacks found in the previous protocols is proposed in this paper.

\section{THE PROPOSED RFID AUTHENTICATION PROTOCOL}

In this section, we explain the proposed protocol in detail.

\section{Assumptions}

We present a lightweight RFID mutual authentication protocol, which operates under the following assumptions: 
- The communication between the reader and the tag is initiated by the reader, i.e. tags are passive.

- The reader contacts the tag through a wireless channel which is susceptible to different attacks such as location tracking, replay attacks, DoS attacks and impersonation attacks.

- The communication channel between the reader and server is secure.

- The tag's data are stored in non-volatile memory such as EEPROM or Flash memory, where they can be updated.

- All the operations in the tag are atomic i.e. either all of the operations or none are processed. If the attacker kills the electromagnetic field between the reader and tag or simply the tag walks away from the reader's signal, the tag will execute all the computations simultaneously or not at all.

- We assume that the proposed protocol supports a multiple readers scenario, all connected to a central server, so that a tag can be read in many different locations.

\section{Protocol behaviour}

The proposed protocol has the following main features:

- The proposed protocol uses random numbers in an attempt to prevent location tracking and replay attacks.

- The server stores both the old and the new values of the data in order to prevent desynchronization attacks.

- After a successful authentication between the server and tag, both parties update their values to be used in the next transaction.

\section{Notation}

The notation used in the proposed protocol is presented below:

1) The notation related to the server database is,

- ID old: The tag's old ID

- $\quad \mathrm{ID}_{\text {new }}$ : The tag's new ID

- $\quad \mathrm{K}_{\text {old }}$ : The old secret key

- $\quad \mathrm{K}_{\text {new }}$ : The new secret key

2) The notation related to the tag is,

- $T_{\mathrm{i}}$ : The $\mathrm{i}^{\text {th }}$ tag of the RFID system, where $1 \leq \mathrm{i} \leq \mathrm{N}$

- ID: The tag's ID, shared with the server's ID old $_{\text {or }} \mathrm{ID}_{\text {new }}$

- K: The tag's secret key shared with the server's $\mathrm{K}_{\text {old }}$ or $\mathrm{K}_{\text {new }}$

3) Other notation used in the proposed protocol is,
- $\quad$ x: The value kept as either new or old to show whether the tag uses the old or new values of ID and $\mathrm{K}$

- R1: A pseudo random number generated by the reader

- R2: A pseudo random number generated by the tag and serving as a temporary secret for the tag

- H: A hash function, h: $\{0,1\}^{*} \rightarrow\{0,1\}^{\mathrm{L}}$, where $\mathrm{L}$ is equal to the length of the data

- $\mathrm{A} \oplus \mathrm{B}$ : Message $\mathrm{A}$ is XORed with message $B$

- $\quad$ A || B: Message A is concatenated with message $\mathrm{B}$

- $A \leftarrow B$ : The value of $A$ is updated to that of $\mathrm{B}$

- $\quad \mathrm{j}$ : The transaction number

- $\quad \mathrm{N}$ : The number of tags managed by the server

\section{Protocol description}

The scheme consists of two processes namely initialization, and authentication.

1) Initialisation Process: This stage only occurs during manufacturing when the manufacturer assigns the initial values in the server, and in the tag. The initialisation process is summarised below:

- The server assigns random values for each tag it manages to ( $\left.\mathrm{ID}_{\text {new }}, \mathrm{K}_{\text {new }}\right)$ in the server and (ID, K) in the tag.

- Initially, (ID old, $\mathrm{K}_{\text {old }}$ ) in the server is set to null.

2) Authentication Process: The authentication process is shown in Table 1.

- Reader: The reader generates a random number $\mathrm{R} 1$ of $\mathrm{L}$ bits and sends it to the tag.

- Tag:

- The tag generates a random number R2 of $\mathrm{L}$ bits as a temporary secret for the session, and computes:

$\mathrm{HID}=\mathrm{H}(\mathrm{ID} \| \mathrm{R} 1)$,

$\mathrm{M} 1=\mathrm{H}(\mathrm{K}\|\mathrm{R} 1\| \mathrm{R} 2)$,

$\mathrm{M} 2=\mathrm{ID} \oplus \mathrm{R} 2$

- $\quad$ The tag sends HID, M1 and M2 to the reader.

- $\quad$ Reader: The reader sends R1, HID, M1, and M2 to the server.

- Server:

- For all the stored IDs, the server computes $\mathrm{H}(\mathrm{ID} \| \mathrm{R} 1)$ until it finds a match with the received value of HID: 
- If there is a match in $\mathrm{ID}_{\text {new }}$, then the server marks $\mathrm{x}=$ new. The server retrieves data $\left(\mathrm{ID}_{\text {new }}\right.$, $\mathrm{K}_{\text {new }}$ ), extracts $\mathrm{R} 2$ i.e. $\mathrm{R} 2=\mathrm{ID}_{\text {new }}$ $\oplus \mathrm{M} 2$, and re-computes $\mathrm{M} 1$ i.e. $\mathrm{M}^{\prime} 1=\mathrm{H}\left(\mathrm{K}_{\text {new }}\|\mathrm{R} 1\| \mathrm{R} 2\right)$ to authenticate the tag.

- If there is a match in ID $_{\text {old }}$, then the server marks $\mathrm{x}=\mathrm{old}$, retrieves the data ( $\mathrm{ID}_{\text {old }}, \mathrm{K}_{\text {old }}$ ), extracts $\mathrm{R} 2$ i.e. $\mathrm{R} 2=\mathrm{ID}_{\text {old }} \oplus \mathrm{M} 2$ and recalculates $\mathrm{M}^{\prime} 1=\mathrm{H}\left(\mathrm{K}_{\text {old }}\|\mathrm{R} 1\| \mathrm{R} 2\right)$ to authenticate the tag.

- $\quad$ The server computes $\mathrm{M} 3=\mathrm{H}\left(\mathrm{ID}_{\mathrm{x}}\right.$ \| $\mathrm{K}_{\mathrm{x}}$ || R1 || R2), and transmits it to the reader.

- The server updates the data as follows:

If $x=$ new, where ID is found in $\mathrm{ID}_{\text {new }}$

$$
\begin{aligned}
& \mathrm{ID}_{\text {new }}^{\mathrm{j}+1} \leftarrow \mathrm{H}\left(\mathrm{ID}_{\text {new }}^{\mathrm{j}}\right) \\
& \mathrm{ID}_{\text {old }}^{\mathrm{j}+1} \leftarrow \mathrm{ID}_{\text {new }}^{\mathrm{j}} \\
& \mathrm{K}_{\text {new }}^{\mathrm{j}+1} \leftarrow \mathrm{H}^{\mathrm{j}}\left(\mathrm{K}_{\text {new }}^{\mathrm{j}} \oplus \mathrm{ID}_{\text {new }}^{\mathrm{j}+1}\right) \\
& \mathrm{K}_{\text {old }}^{\mathrm{j}+1} \leftarrow \mathrm{K}_{\text {new }}^{\mathrm{j}}
\end{aligned}
$$

Else if $\mathrm{x}=\mathrm{old}$, where ID is found in $\mathrm{ID}_{\text {old }}$ :

No updates

If there is no match in $\mathrm{ID}_{\text {new }}$ and $\mathrm{ID}_{\text {old }}$ or $\mathrm{M}^{\prime} 1 \neq \mathrm{M} 1$ or $\mathrm{M}^{\prime} 2 \neq \mathrm{M} 2$, then the server sends an end session message to the reader to terminate the session.

- $\quad$ Reader: Once the reader receives M3, it sends M3 to the tag.

- Tag: The tag determines whether the received value of M3 is equal to $H$ (ID \| $\mathrm{K}\|\mathrm{R} 1\| \mathrm{R} 2$ ). If there is a match, the tag authenticates the server and updates its values to:

$$
\begin{aligned}
& \mathrm{ID}^{\mathrm{j}+1} \leftarrow \mathrm{H}\left(\mathrm{ID}^{\mathrm{j}}\right) \\
& \mathrm{K}^{\mathrm{j}+1} \leftarrow \mathrm{H}\left(\mathrm{K}^{\mathrm{j}} \oplus \mathrm{ID}^{\mathrm{j}+1}\right)
\end{aligned}
$$

If the check fails or M3 is not received, the tag keeps the current values unchanged.

\section{Protocol analysis}

In this section, we analyse the proposed protocol in terms of informal and formal analysis using a privacy model, CasperFDR and AVISPA. Finally, we present the expected performance measurement.

\subsection{Informal security analysis of the protocol}

In Table 2 We compared our protocol with the related research work against the main requirements shown in Section 2. Our proposed protocol provides the following goals:

Our proposed protocol provides the following goals:

1) Tag anonymity: The tag stores two values, namely (ID, K) that supposed to be secret and not revealed to any entity except the legitimate server. Only the legitimate server that has information related to the tag can extract these values.

2) Tag location privacy (untraceability): In the proposed protocol, the tag's responses are changed with new updated values and fresh random numbers, thus the attacker will obtain new responses every time he eavesdrops on a session. Moreover, if the previous authentication session failed and the tag's data remain unchanged, HID, M1 and $\mathrm{M} 2$ responses will change due to the existence of new fresh random numbers.

3) Resistance to replay attack: The proposed protocol utilises a challenge-response scheme, where each party maintains a set of random numbers it has seen from previous protocol run to avoid repeated random numbers. Thus, when the tag or server detects repeated random numbers, it will terminate the session.

4) Resistance to desynchronisation attack: In the proposed protocol, the desynchronisation attack is avoided via storing the previous values of the data (ID ${ }_{\text {old }}, \mathrm{K}_{\mathrm{old}}$ ), and thus reach synchronization. Moreover, the server does not update its data when there is a match in (ID old, $\mathrm{K}_{\text {old }}$ ), it keeps the stored data the same. Thus when the attacker blocks M3 more than once respectively, the tag's data (ID, K) will still match the server's data (ID old, $\mathrm{K}_{\text {old }}$ ).

5) Resistance to tag impersonation attack: To impersonate the tag, the attacker must be able to compute a valid response (HID, M1 and M2) to a server query. However, it is hard to compute such responses without knowledge of ID, and K.

6) Resistance to server impersonation attack: To impersonate the server, the attacker must be able to compute a valid response (M3). However, it is hard to compute such responses without knowledge of $\mathrm{ID}_{\mathrm{x}}, \mathrm{K}_{\mathrm{x}}$, and R2. 


\subsection{Privacy analysis}

The researchers have proposed number of privacy models to evaluate the privacy of the RFID protocols such as [21, 35]. The model in [21] is summarised as follows: An adversary (A) controls the communication channel between a tag $(\mathrm{T})$ and a reader $(\mathrm{R})$ by interacting either passively or actively with them. The adversary can run the following queries:

- Execute $(R, T, i)$ query: The adversary can passively eavesdrop on a session (i) and obtain access to the exchanged messages between $R$ and $\mathrm{T}$.

- Send $(U, V, m, i)$ query: The adversary can perform active attacks by impersonating an entity such as $\mathrm{V} \in \mathrm{T}$ and sends a message (m) to entity $U \in R$ during session (i). Also, he can alter or block some of the exchanged messages.

- Corrupt $\left(T, K^{\prime}\right)$ query: The attacker can physically access the tag's memory $\mathrm{T}$ and read the tag's secret value $\left(\mathrm{K}^{\prime}\right)$.

- Test $\left(i, T_{0}, T_{l}\right)$ query: This query is used to define the untraceability test. When this query is invoked for session (i), a random bit b2 $\in\{0$, $1\}$ is generated and then, $A$ is given $T_{b} \in\left\{T_{0}\right.$, $\mathrm{T}_{1}$ \}. Informally, $\mathrm{A}$ wins if he can guess the bit b.

Untraceable privacy (UPriv) is defined as a game (g) played by the adversary (A) and a collection of the reader and the tag instances. The game consists of three phases:

1) Learning phase: The adversary (A) can send the Execute, Send, and Corrupt queries to any random $\mathrm{T}_{0}$ and $\mathrm{T}_{1}$ tags.

2) Challenge phase: The adversary (A) is given a tag $\mathrm{T}_{\mathrm{b}} \in\left\{\mathrm{T}_{0}, \mathrm{~T}_{1}\right\}$, and sends any Execute, and Send queries to $\mathrm{T}_{\mathrm{b}}$.

3) Guess phase: A terminates the game and outputs a bit $b_{0}$, which is its guess of the value of $b$.

The success of A in winning ( $\mathrm{g}$ ) and breaking the untraceability privacy (UPriv) is achieved in terms of A's advantage in distinguishing whether A received $\mathrm{T}_{0}$ or $\mathrm{T}_{1}$, i.e. it correctly guessing $\mathrm{b}$. This is denoted by $A d v_{A}^{U P r i v} \mathrm{k}$, where $\mathrm{k}$ is the security parameter. Now, we will evaluate the privacy of our proposed protocol using this model. We found that the adversary cannot invade the privacy of the tag and trace its location as shown below:

1) Learning phase: The adversary eavesdrops a valid session between $R$ and $T_{0}$. He sends the Execute command and then maintains the following values, which are sent :

\author{
$\mathrm{R} 1, \mathrm{HID}=\mathrm{H}\left(\mathrm{ID}_{0} \| \mathrm{R} 1\right)$ \\ $\mathrm{M} 1=\mathrm{H}\left(\mathrm{K}_{0}\|\mathrm{R} 1\| \mathrm{R} 2\right)$ \\ $\mathrm{M} 2=\mathrm{ID}_{0} \oplus \mathrm{R} 2$
}

2) Challenge phase: $A$ is given a tag $T_{b} \in\left\{T_{0}\right.$, $\left.\mathrm{T}_{1}\right\}$ randomly. He starts a new session with $\mathrm{T}_{\mathrm{b}}$ by impersonating the reader and sends $\mathrm{R} 1$ to $\mathrm{T}_{\mathrm{b}}$ within the Send query and terminates the session. $\mathrm{T}_{\mathrm{b}}$ responds can be:

$$
\begin{aligned}
& \mathrm{HID}=\mathrm{H}\left(\mathrm{ID}_{\mathrm{b}} \| \mathrm{R} 1\right) \\
& \mathrm{M} 1=\mathrm{H}\left(\mathrm{K}_{\mathrm{b}}\|\mathrm{R} 1\| \mathrm{R} 2^{\prime}\right) \\
& \mathrm{M} 2=\mathrm{ID}_{\mathrm{b}} \oplus \mathrm{R} 2^{\prime}
\end{aligned}
$$

However, A will not be able to guess the correct tag (bit b) as the received messages M1 and M2 contain a random number (R2) generated by the tag, which changes in every session, and not known to the adversary. Moreover, regarding HID message, if the tag encounters a repeated random number such as $\mathrm{R} 1$, it will terminate the session.

\subsection{Formal Analysis of the Protocol Using CasperFDR}

CasperFDR is a compiler that takes a high level description of the protocol and analyses the protocol description against the stated specification to show whether the protocol meets the main requirements.

Previously, researchers $[36,37]$ have attempted to model their protocol using a Communication Sequential Process (CSP) and a Failure-Divergence Refinement (FDR). CSP is a language for specifying the protocol behaviour. The generated CSP file is analysed by FDR. FDR is a model checker that analyses a protocol and verifies the given specifications. Gavin Lowe has developed

CasperFDR tool [19], which takes a high level description of the protocol together with its security requirements and produces a CSP code checked and verified by FDR.

CasperFDR is used to verify the authentication and secrecy requirements of the protocol. Authentication has two forms of specification namely Agreement and NonInjectiveAgreement.

Agreement means if Bob meets the Agreement specification, he confirms that Alice has run the same protocol, and agreed on the exchanged values. For example Agreement (T, S, [R1, R2, ID, K]) means that the tag is authenticated to the server and both parties agreed on the data values (R1,R2, ID, $\mathrm{K}$ ), and it is one to one relationship i.e. each run of the tag corresponds to a unique run of the server. Another form of authentication specification is NonInjectiveAgreement which differs from Agreement whereas each run can be repeated and overlap. 
Table 1. Goals and requirements comparison

\begin{tabular}{|l|l|l|l|l|l|l|l|l|l|l|l|l|l|l|}
\hline & WP & WP2 & OP & MP & DP & DucP & CP & SG & PP & SP2 & YP & YoonP & HP & Sec.4 \\
\hline $\begin{array}{l}\text { Tag } \\
\text { anonymity }\end{array}$ & $\sqrt{ }$ & $\sqrt{ }$ & $\sqrt{ }$ & $\sqrt{ }$ & $\sqrt{ }$ & $\sqrt{ }$ & $\sqrt{ }$ & $\sqrt{ }$ & $\sqrt{ }$ & $\sqrt{ }$ & $\sqrt{ }$ & $\sqrt{ }$ & $\sqrt{ }$ & $\sqrt{ }$ \\
\hline $\begin{array}{l}\text { Location } \\
\text { privacy }\end{array}$ & $\times$ & $\sqrt{ }$ & $\sqrt{ }$ & $\sqrt{ }$ & $\times$ & $\sqrt{ }$ & $\times$ & $\sqrt{ }$ & $\sqrt{ }$ & $\sqrt{ }$ & $\sqrt{ }$ & $\times$ & $\sqrt{ }$ & $\sqrt{ }$ \\
\hline $\begin{array}{l}\text { Replay } \\
\text { attacks }\end{array}$ & $\times$ & $\times$ & $\times$ & $\sqrt{ }$ & $\sqrt{ }$ & $\times$ & $\sqrt{ }$ & $\sqrt{ }$ & $\sqrt{ }$ & $\sqrt{ }$ & $\sqrt{ }$ & $\times$ & $\sqrt{ }$ & $\sqrt{ }$ \\
\hline $\begin{array}{l}\text { Denial } \\
\text { service } \\
\text { attacks }\end{array}$ & $\sqrt{ }$ & $\sqrt{ }$ & $\sqrt{ }$ & $\sqrt{ }$ & $\times$ & $\times$ & $\times$ & $\times$ & $\times$ & $\times$ & $\sqrt{ }$ & $\sqrt{ }$ & $\times$ & $\sqrt{ }$ \\
\hline $\begin{array}{l}\text { Tag } \\
\text { impersonation } \\
\text { attacks }\end{array}$ & $\times$ & $\times$ & $\sqrt{ }$ & $\sqrt{ }$ & $\sqrt{ }$ & $\times$ & $\times$ & $\sqrt{ }$ & $\sqrt{ }$ & $\sqrt{ }$ & $\times$ & $\times$ & $\times$ & $\sqrt{ }$ \\
\hline $\begin{array}{l}\text { Server } \\
\text { impersonation } \\
\text { attacks }\end{array}$ & $\times$ & $\times$ & $\times$ & $\times$ & $\times$ & $\times$ & $\times$ & $\sqrt{ }$ & $\times$ & $\sqrt{ }$ & $\times$ & $\times$ & $\times$ & $\sqrt{ }$ \\
\hline
\end{tabular}

Table1. The proposed protocol authentication process

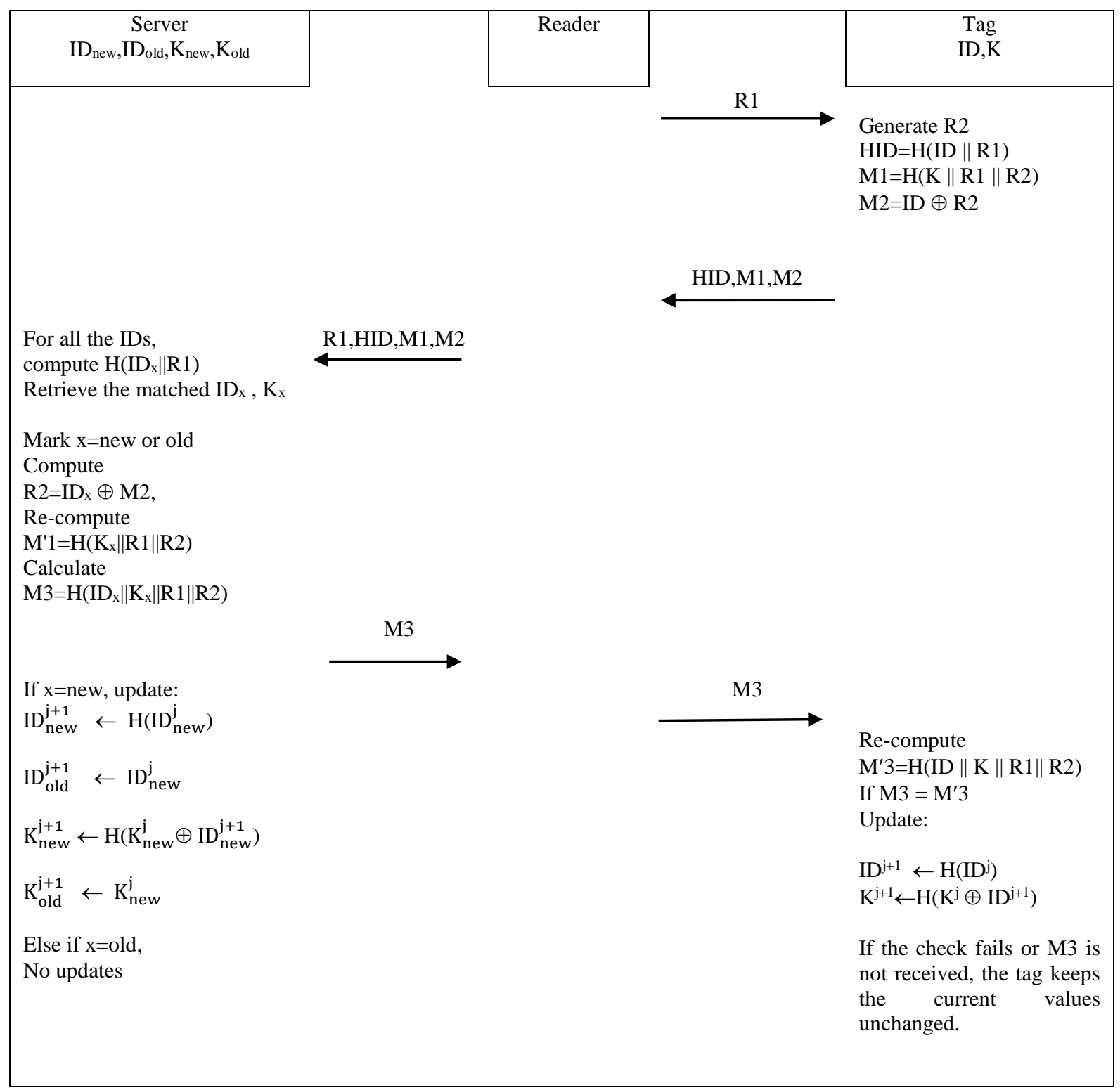


Similarly secrecy has two forms of specification namely Secret and StrongSecret. Secret checks whether the intruder could know the secret value at the end of the protocol. While, StrongSecret checks whether the intruder can obtain the secret value before the protocol finishes its run.

CasperFDR checks the authentication and secrecy specifications by examining the associated events. In other words, Running and Commit events are attached to the authentication specifications. The server and tag are both depicted in CasperFDR as CSP processors. When the tag sends (HID, M1, M2, $\mathrm{R} 1)$ to the server, the server performs the Running event, which means that the server starts running the protocol apparently with the tag. Then, the tag performs the Commit event when it receives the server's reply M3, which means that the tag has finished a run of the protocol with the server [38].

CasperFDR checks the secrecy specifications via an event called Claim_Secret, which is performed by both parties. When the tag receives the server's message (M3), it performs Claim_Secret to ensure that (ID, K, R2) are secret [38]. This process is shown in Figure 1. For simplicity, we treat the server as a reader and a database.

\subsubsection{The proposed protocol requirement illustration in CasperFDR}

Figure 1 shows how the main requirements are achieved in CasperFDR (the numbers in the figure presents the same goals shown below). The following goals are achieved as follow:

1) Mutual authentication: Before the tag sends M1 and M2, it performs Running.T.S.[ID, K,R1,R2] event, which means the tag starts a run of the protocol, apparently with the server agreeing on data. Later, the server will perform Commit.S.T.[ID, K,R1,R2] event at the end of its part of the protocol, which means the server has finished the protocol with the tag agreeing on the received data. Similarly, before the server sends M3, it performs Running.S.T.[ID, $K, R 1, R 2]$, and when the tag receives M3 it performs Commit.T.S.[ID, K,R1,R2].

2) Resistance to impersonation attack: The server performs a Running event such as Running.S.T.ID.R2, which means that the server starts a run of the protocol, apparently with the tag, agreeing on ID and $\mathrm{R} 2$ in $\mathrm{M} 2$. Then, the server performs Running.S.T.K.RI.R2, which means that the server agrees on K, R1 and R2 in M1. Later, the tag will perform the Commit.T.S.ID.R2 and Commit.T.S.K.R1.R2 events at the end of its part of the protocol, which means that the tag has finished the protocol with the server agreeing on the values of ID, K, R1 and $\mathrm{R} 2$.

3) Tag anonymity is depicted as Claim_Secret.T.S.ID and Claim_Secret.T.S.K and Claim_Secret.T.S.R2 events, which means that the three values of ID, K and R2 should be kept secret between the tag and the server.

4) Resistance to replay attack is illustrated as a scenario where the tag is engaging in the protocol twice. The tag firstly runs the protocol with the server, and the intruder obtains R1. Then, the intruder runs the protocol with the same tag and resends R1 to the tag. Therefore, in our protocol the tag will not perform the Commit event as it received the same random number $\mathrm{R} 1$. Similarly, if the server engages in the protocol run by receiving duplicate messages from the intruder or the tag, it will not perform the Running event.

We prepared the CasperFDR script to show some indicative results if there is an attack on the protocol or not. The script is shown in Appendix A. The section \#Specification, specifies the security and authentication requirements of the protocol, the lines Secret (T, K, $[S])$, Secret (T, ID, $[S])$, and Secret (T, $R 2$, $[S]$ ), indicate that the values of $\mathrm{K}, \mathrm{ID}$, and $\mathrm{R} 2$ should only be known by the tag (T) and legitimate server (S). The lines starting with Agreement are for providing authentication for instance, Agreement ( $T$, $S, \quad[R 1, R 2, I D, K])$ means that the tag is authenticated to the server using the data values (R1, $\mathrm{R} 2$, ID, K).

In addition, in the \#Intruder information section, the intruder is defined to be Mallory, who can take a full control of the session; he can impersonate any entity in the protocol, generate a random number, read the messages transmitted in the network, intercept, analyse, and/or modify messages.

CasperFDR did not find any feasible attacks on the proposed protocol.

\subsection{Formal analysis of the protocol using AVISPA}

In addition to using CasperFDR for formally analyzing the proposed protocol, we presented our protocol using a High Level Protocol Specification Language (HLPSL), a specification language for formalizing protocols [20]. This language is then translated with the Automated Validation of Internet Security Protocols and Applications (AVISPA) model checker tool by using a translator called HLPSL2IF and four different integrated verification 
backends called: On the Fly Model Checker(OFMC) [39], Constraint-Logic based Attack Searcher (CLAtSe) [40], SAT based Model-Checker (SATMC) [41] and Tree Automata based Protocol Analyser (TA4SP) [42]. These backends implement a variety of automatic analysis techniques to check the main goals of the protocol such as secrecy and authentication.

In these backends, the intruder is modelled via using the channel(dy) which stands for the DolevYao intruder model [43]. Under this model, the intruder has full control over the network, such that all messages sent by agents can be eavesdropped by the intruder. Moreover, the intruder may intercept, analyse, modify messages, and/or send any message he/she composes to other agents pretending to come from a legitimate agent.

In AVISPA, there are three roles. Firstly, basic role, which defines the agent who runs the protocol and the initial information and parameters the agent holds. Secondly, in the composition role, we describe the sessions of the protocol by specifying how the agents interact with each other. A top-level role environment role contains global constants and a composition of one or more sessions, where the intruder may play some roles as a legitimate user. Moreover, environment role shows what knowledge the intruder initially has.

The main requirements and goals are declared in a section called goal. There are two main goals in AVISPA namely secrecy and authentication. Secrecy is modelled via the goal predicate secret Authentication is modelled by means of the goal predicates witness and request.

The script is shown in Appendix B. To elaborate the script, there are two agents namely a server and a tag. We assume that the server acts as a reader and a database. The server and tag shares a symmetric key $(\mathrm{K})$ and they both utilise a hash function $(\mathrm{H})$ for the calculation of the messages. The tag ID, $\mathrm{ID}_{\text {old }}$ and random numbers $\mathrm{R} 1$ and $\mathrm{R} 2$ are defined as text. ID defines $I_{\text {new. }}$ R1 and R2 are freshly generated using the new function.

In the script, authentication is achieved via witness and request goals i.e. witness $(S, T$, trid, $I D)$ which declares that agent $\mathrm{S}$ asserts to be the peer of agent $\mathrm{T}$, agreeing on the value ID, trid is the name of the ID authentication shown in the goal section. request(T,S,trid,Auth') can be read as "agent $\mathrm{T}$ accepts the value Auth1 and now relies on the guarantee that agent $\mathrm{S}$ exists and agrees with it on this value".

Regarding secrecy, secret $(I D, i d, T, S)$ means that the value of ID should be a secret between agents $T$ and $\mathrm{S}$, and id is the name of the secret term, which is defined in the goal section.

In the environment role, the intruder is identified and we assume that the intruder knows the other agents (tag and server), keys he shares with the agents, and hash function.
Our protocol script has been analysed by the OFMC and CL-AtSe backends as they support an exclusive-OR properties. The results show that the protocol is safe. The other backends SATMC and TA4SP do not support an exclusive-OR property that is why the result shows inconclusive.

\subsection{Performance analysis}

In this section, we conduct a comparative analysis of the performance cost regarding storage cost, and communication cost.

a) Storage cost: Due to the limitation of tag memory, the tag should store minimum amount of data. In the proposed protocol, the tag stores two values in a rewritable flash memory namely (ID, K), as they change in different authentication sessions, each of which has a length of 224 bits. Since the tag's memory can store 1 Kilobyte of data, in our protocol the tag securely stores $224 * 2=448$ bits in the memory. Additional tag memory is necessary in our protocol to store a list of random numbers received from previous queries, for example by adding extended on-chip non-volatile memory on the RFID tags.

b) Communication cost: In the proposed protocol, the tag sends three messages (HID, M1 and M2) in order to be successfully authenticated. A total of 672 bits are sent over the channel as the length of one message is 224 bits. Hence, it provides a relatively low communication cost.

\section{Conclusion}

In this paper we presented a lightweight RFID mutual authentication protocol that based on the strength found in previous protocols and prevent their deficiencies. The protocol has been informally and formally analysed using formal methods. Firstly, based on the informal analysis, the results demonstrate that the protocol performs better than the selected protocols and offers immunity against a broad range of attacks. Secondly, the privacy of the tag's data is evaluated via the privacy model, which showed that the tag's data cannot be traced or compromised. Thirdly, the secrecy and authentication requirements have been analysed via CasperFDR and AVISPA formal tools. CasperFDR and AVISPA did not show any feasible attacks. Finally, we conducted a performance comparative analysis in terms of storage, communication costs and server scalability, and we concluded that the proposed protocol is compatible with the RFID systems requirements. 


\section{Acknowledgment}

This research was supported by the Ministry of Higher Education and King Khaled University in Saudi Arabia.

\section{References}

[1] K. Finkenzeller and R. Waddington, RFID handbook: radio-frequency identification fundamentals and applications. Wiley New York, 1999.

[2] A. Juels, "RFID security and privacy: A research survey," Selected Areas in Communications, IEEE Journal on, vol. 24, no. 2, pp. 381-394, 2006.

[3] K. Finkenzeller, "RFID handbook: Fundamentals and applications in contactless smart cards and identification," $2^{\text {nd }}$ ed. New York, USA, John Wiley, 2003.

[4] J. Li, Y. Wang, B. Jiao, and Y. Xu, "An authentication protocol for secure and efficient RFID communication," in Logistics Systems and Intelligent Management, 2010 International Conference on, vol. 3. IEEE, 2010, pp. 1648-1651.

[5] P. Pessl and M. Hutter, "Pushing the limits of SHA-3 hardware implementations to fit on RFID," in Cryptographic Hardware and Embedded Systems-CHES 2013. Springer, 2013, pp. 126-141.

[6] B. Song and C. Mitchell, "RFID authentication protocol for low-cost tags," in Proceedings of the first ACM conference on Wireless network security. ACM, 2008, pp. 140-147.

[7] H. Chien and C. Chen, "Mutual authentication protocol for RFID conforming to EPC Class 1 Generation 2 Standards," Computer Standards Interfaces, vol. 29, no. 2, pp. 254-259, 2007.

[8] D. Duc, H. Lee, and K. Kim, "Enhancing security of EPCglobal Gen-2 RFID against traceability and cloning," in Symposium on Cryptography and Information Security. The Institute of Electronics, Information and Communication Engineers, 2006.

[9] T. Dimitriou, "A lightweight RFID protocol to protect against traceability and cloning attacks," in Security and Privacy for Emerging Areas in Communications Networks, 2005. SecureComm 2005. First International Conference on. IEEE, 2005, pp. 59-66. [10] M. Shemaili, C. Yeun, and M. Zemerly, "RFID lightweight mutual authentication using shrinking generator," in Internet Technology and Secured Transactions, 2009. ICITST 2009. International Conference for, 2009, pp. 1-6.

[11] G. Poulopoulos, K. Markantonakis, and K. Mayes, "A Secure and Efficient Mutual Authentication Protocol for Low-Cost RFID Systems," in Availability, Reliability and Security, 2009. ARES'09. International Conference on. IEEE, 2009, pp. 706-711.

[12] S. Weis, S. Sarma, R. Rivest, and D. Engels, "Security and privacy aspects of low-cost radio frequency identification systems," Security in pervasive computing, pp. 50-59, 2004.

[13] M. Ohkubo, K. Suzuki, S. Kinoshita et al., "Cryptographic approach to "privacy-friendly" tags," in
RFID Privacy Workshop, vol. 82. MIT, Cambridge, MA, 2003.

[14] S. Weis, "Security and privacy in radiofrequency identification devices," Ph.D. dissertation, Massachusetts Institute of Technology, 2003.

[15] T. Yeh, Y. Wang, T. Kuo, and S. Wang, "Securing RFID systems conforming to EPC Class 1 Generation 2 Standard," Expert Systems with Applications, vol. 37, no. 12, pp. 7678-7683, 2010.

[16] E. Yoon, "Improvement of the securing RFID systems conforming to EPC Class 1 Generation 2 Standard," Expert Systems with Applications, vol.39, no. 1, pp. 1589-1594, 2012.

[17] Y. HanataniI, M. Ohkubo, S. Matsuo, K. Sakiyama, and K. Ohta, "A study on computational formal verification for practical cryptographic protocol: The case of synchronous RFID authentication," Financial Cryptography and Data Security, pp. 70-87, 2012.

[18] D. N. Duc and K. Kim, "Defending RFID authentication protocols against DoS attacks," Computer Communications, vol. 34, no. 3, pp. 384-390, 2011.

[19] G. Lowe, "Casper: A compiler for the analysis of security protocols," in Computer Security Foundations Workshop, 1997. Proceedings., 10th. IEEE, 1997, pp. 1830.

[20] A. Armando, D. Basin, Y. Boichut, Y. Chevalier, L. Compagna, J. Cuéllar, P. H. Drielsma, P.-C. Héam, O. Kouchnarenko, J. Mantovani et al., "The AVISPA tool for the automated validation of internet security protocols and applications," in Computer Aided Verification. Springer, 2005, pp. 281-285.

[21] K. Ouafi and R. Phan, "Privacy of recent RFID authentication protocols," in Information Security Practice and Experience, ser. Lecture Notes in Computer Science, L. Chen, Y. Mu, and W. Susilo, Eds. Springer Berlin Heidelberg, 2008, vol. 4991, pp. 263-277. [Online]. Available: http://dx.doi.org/10.1007/978-3-540-791041_19

[22] A. Mitrokotsa, M. Rieback, and A. Tanenbaum, "Classifying RFID attacks and defenses," Information Systems Frontiers, vol. 12, no. 5, pp. 491-505, 2010.

[23] H. Kim, J. Oh, J. Choi, and J. Kim, "The Vulnerabilities Analysis and Design of the Security Protocol for RFID System," in Computer and Information Technology, 2006. CIT'06. The Sixth IEEE International Conference on. IEEE, 2006, pp. 152-152.

[24] B. Song, "RFID authentication protocols using symmetric cryptography," Ph.D. dissertation, Royal Holloway, University of London, 2009.

[25] G. Avoine, E. Dysli, and P. Oechslin, "Reducing time complexity in RFID systems," in Selected Areas in Cryptography. Springer, 2006, pp. 291-306.

[26] D. Molnar and D. Wagner, "Privacy and Security in Library RFID: issues, Practices, and Architectures," in Proceedings of the 11th ACM conference on Computer and communicationssecurity. ACM, 2004, pp. 210-219.

[27] P. Peris-Lopez, J. Hernandez-Castro, J. EstévezTapiador, and A. Ribagorda, "LMAP: A real lightweight mutual authentication protocol for low-cost RFID tags," in Proc. of 2nd Workshop on RFID S

[28] D. Coppersmith, H. Krawczyk, and Y. Mansour, "The shrinking generator," in Advances in Cryptology CRYPTO '93, 13th Annual International Cryptology Conference, Santa Barbara, California, USA, August 22- 
26, 1993, Proceedings, ser. Lecture Notes in Computer Science, vol. 773. Springer, 1993, pp. 22-39.

[29] S. Cai, Y. Li, T. Li, and R. Deng, "Attacks and improvements to an RFID mutual authentication protocol and its extensions," in Proceedings of the second ACM conference on Wireless network security. ACM, 2009, pp. $51-58$.

[30] P. Rizomiliotis, E. Rekleitis, and S. Gritzalis, "Security analysis of the Song-Mitchell authentication protocol for low-cost RFID tags," Communications Letters, IEEE, vol. 13, no. 4, pp. 274-276, 2009.

[31] S. Abughazalah, K. Markantonakis, and K. Mayes, "A vulnerability in the Song authentication protocol for low-cost RFID tags," in Security and Privacy Protection in Information Processing Systems, ser. IFIP Advances in Information and Communication Technology, L. Janczewski, H. Wolfe, and S. Shenoi, Eds. Springer Berlin Heidelberg, 2013, vol. 405, pp. 102-110.

[32] S. Abughazalah, K. Markantonakis, and K. Mayes, "Desynchronisation attacks on an RFID mutual authentication protocol," to be published.

[33] M. Habibi, M. Aref, and D. Ma, "Addressing flaws in RFID authentication protocols," in Progress in Cryptology-INDOCRYPT 2011. Springer, 2011, pp. 216235

[34] J. Hernandez-Castro, P. Peris-Lopez, M. Safkhani, N. Bagheri, and M. Naderi, "Another fallen hash-based RFID authentication protocol," Information Security Theory and Practice. Security, Privacy and Trust in Computing Systems and Ambient Intelligent Ecosystems, pp. 29-37, 2012.

[35] G. Avoine, "Adversarial model for radio frequency identification." IACR Cryptology ePrint Archive, vol. 2005, p. 49, 2005.

[36] G. Lowe, "Breaking and fixing the NeedhamSchroeder public-key protocol using FDR," in Tools and Algorithms for the Construction and Analysis of Systems. Springer, 1996, pp. 147-166.

[37] G. Lowe and B. Roscoe, "Using CSP to detect errors in the TMN protocol," Software Engineering, IEEE Transactions on, vol. 23, no. 10, pp. 659-669, 1997.

[38] A. Alshehri and S. Schneider, "Formally defining nfc m-coupon requirements, with a case study," The 5th International Workshop on RFID Security and Cryptography 2013 (RISCï $i 1 / 213$ ), Internet Technology and Secured Transactions, pp. 58-64, December 2013.

[39] D. Basin, S. Modersheim, and L. Vigano, "An on-the-fly model-checker for security protocol analysis," in Computer Security " ESORICS 2003, ser. Lecture Notes in Computer Science, E. Snekkenes and D. Gollmann, Eds. Springer Berlin Heidelberg, 2003, vol. 2808, pp. 253-270. [Online]. Available: http://dx.doi.org/10.1007/978-3-54039650-5_15

[40] M. Turuani, "The CL-ATSE protocol analyser," in Term Rewriting and Applications, ser. Lecture Notes in Computer Science, F. Pfenning, Ed. Springer Berlin Heidelberg, 2006, vol. 4098, pp. 277-286. [Online]. Available: http://dx.doi.org/10.1007/11805618_21

[41] K. McMillan, "Interpolation and SAT-based model checking," in Computer Aided Verification, ser. Lecture Notes in Computer Science, J. Hunt, WarrenA. and F. Somenzi, Eds. Springer Berlin Heidelberg, 2003, vol. 2725, pp. 1-13. [Online]. Available: http://dx.doi.org/10.1007/978-3-540-45069-6_1

[42] Y. Boichut, P. Héam, O. Kouchnarenko, and F. Oehl, "Improvements on the Genet and Klay technique to automatically verify security protocols," in Proc. AVIS, vol. 4, 2004.

[43] D. Dolev and A. Yao, "On the security of public key protocols," Information Theory, IEEE Transactions on, vol. 29, no. 2, pp. 198-208, 1983.

\section{Appendix A CasperFDR Script}

\#Free variables

$\mathrm{T}$ : Agent

$S$ : Server

$\mathrm{R} 1$ : initialSeq

$\mathrm{R} 2$ : Sequence

IDold, ID : SessionID

Kold , K : SessionKey

h: HashFunction

InverseKeys $=($ Kold,Kold $),(\mathrm{K}, \mathrm{K})$,

(IDold,IDold) , (ID , ID)

\#Protocol description

0. $\quad \rightarrow \mathrm{S}: \mathrm{T}$

1. $\mathrm{S} \rightarrow \mathrm{T}: \mathrm{R}$

2a. $T$ - $>\mathrm{S}: \mathrm{R} 2(+) \mathrm{ID}$

2b. T $>$ S : h(K, R1, R2)

[IDold $==$ ID and Kold $==\mathrm{K}$

or ID $==$ ID and $\mathrm{K}==\mathrm{K}$ ]

4. S -> T : h(ID, K, R1, R2)

\#Processes

RESPONDER(T, S, R2, K, ID)

SERVER (S, T, R1, Kold, IDold, K, ID)

\#Actual variables

Tag, Mallory : Agent

ServerDB : Server

Rr1 : initialSeq

$\operatorname{Rr} 2$ : Sequence

R3 : Sequence

IDentityO,IDentityT:SessionID

KeyOld, KeyTag : SessionKey

InverseKeys=(KeyOld,KeyOld),

(KeyTag, KeyTag), (IDentityO,IDentityO),

,(IDentityT,IDentityT)

\#Specification

Aliveness(S, T)

Secret(T, K, [S])

Secret(T, ID, [S])

Secret(T, R2, [S])

Agreement(T, S, [R1, R2, ID,K])

Agreement(S, T, [R1, R2, ID,K])

\#System

RESPONDER(Tag,ServerDB,Rr2,KeyTag,IDentityT)

SERVER(ServerDB, Tag, Rr1, KeyOld, KeyTag

IDentityO, IDentityT)

\#Intruder Information

Intruder $=$ Mallory

IntruderKnowledge $=\{$ Tag,ServerDB,Mallory,R3 $\}$

\section{Appendix B AVISPA Script}

role server(S, T: agent,

$\mathrm{K}:$ symmetric_key,

$\mathrm{H}:$ hash_func,

SND, RCV: channel(dy)) 
played_by $S$ def=

local State: nat,

R1,R2, ID, IDold:text,

Auth1:hash(symmetric_key.text.text),

Kold : symmetric_key

init State $:=0$

transition

0 . State $=0 \wedge R C V($ start $)$

$=\mid>$ State' $:=1$

$\wedge \mathrm{R} 1^{\prime}:=$ new ()

$\wedge \mathrm{SND}\left(\mathrm{R} 1^{\prime}\right)$

1. State $=3 \wedge \mathrm{K}^{\prime}=\mathrm{K} \wedge \mathrm{ID}^{\prime}=\mathrm{ID}$

$\wedge \mathrm{RCV}\left(\mathrm{H}\left(\mathrm{K} \cdot \mathrm{R} 1 \cdot \mathrm{R} 2^{\prime}\right) \cdot \operatorname{xor}\left(\mathrm{R} 2^{\prime}, \mathrm{ID}\right)\right)=\mid>$

State' $:=4 \wedge$ Auth1':= H(K.R1.R2')

$\wedge$ request(S,T,id3,Auth1')

$\wedge \mathrm{SND}(\mathrm{H}(\mathrm{ID} . \mathrm{K} . \mathrm{R} 1 . \mathrm{R} 2 '))$

$\wedge$ witness(S, T, trid, ID)

$\wedge$ witness( $\mathrm{S}, \mathrm{T}$, trk, K)

$\wedge$ ID':= new ()

$\wedge$ IDold' $:=$ new ()

$\wedge \mathrm{K}^{\prime}:=$ new ()

$\wedge$ Kold' $:=$ new ()

1. State $=3 \wedge$ Kold' $=K \wedge$ IDold' $=$ ID

$\wedge \mathrm{RCV}\left(\mathrm{h}\left(\mathrm{K} \cdot \mathrm{R} 1 \cdot \mathrm{R} 2^{\prime}\right) \cdot \mathrm{xor}\left(\mathrm{R} 2^{\prime}, \mathrm{ID}\right)\right)=\mid>$

State' $:=4 \quad \wedge$ Auth1' $:=$ H(Kold.R1.R2')

$\wedge$ request(S,T,id3,Auth1')

$\wedge \mathrm{SND}(\mathrm{H}$ (IDold.Kold.R1.R2'))

$\wedge$ witness(S, T, trid, IDold)

$\wedge$ witness( $S, T$, trk, K)

end role

$\% \% \% \% \% \% \% \% \% \% \% \% \% \% \% \% \% \% \% \% \% \% \%$ role tag( T,S: agent,

K: symmetric_key,

$\mathrm{H}$ : hash func,

SND,RCV: channel(dy))

played_by T def $=$

local State : nat,

R1,R2, ID: text,

Auth : hash(text. text.text)

init State $:=0$

transition

0. State $=0 \wedge R C V\left(R 1^{\prime}\right)$

$=\mid>$ State $^{\prime}:=1$

$\wedge \mathrm{R}^{\prime}:=$ new ()

$\wedge \mathrm{SND}\left(\mathrm{h}\left(\mathrm{K} . \mathrm{R} 1^{\prime} \cdot \mathrm{R} 22^{\prime}\right) . x o r\left(\mathrm{R} 2{ }^{\prime}, \mathrm{ID}\right)\right)$

$\wedge$ witness( $T, S$, trid,ID)

$\wedge$ witness( $T, S$, trk, K)

1. State $=1 \wedge \operatorname{RCV}\left(\mathrm{h}\left(\mathrm{ID} \cdot \mathrm{K} \cdot \mathrm{R} 1^{\prime} . \mathrm{R} 2^{\prime}\right)\right)$

$=\mid>$ State $^{\prime}:=2$

$\wedge$ Auth' := h(ID.K.R1'.R2')

$\wedge$ request(T,S,trid,Auth') $\wedge \operatorname{secret}(\mathrm{ID}, \mathrm{id},\{\mathrm{T}, \mathrm{S}\})$

$\wedge$ secret $(\mathrm{R} 2 ', \mathrm{id} 2,\{\mathrm{~T}, \mathrm{~S}\})$

$\wedge \operatorname{secret}(\mathrm{K}, \mathrm{id} 3,\{\mathrm{~T}, \mathrm{~S}\})$

$\wedge$ ID':=new()

$\wedge \mathrm{K}^{\prime}:=$ new ()

end role

$\% \% \% \% \% \% \% \% \% \% \% \% \% \% \% \% \% \% \% \% \% \% \% \% \%$ role session( T,S : agent,

$\mathrm{K}$ : symmetric_key,

Hash : hash_func)

$\operatorname{def}=$

local SND, RCV: channel (dy)

composition

$\operatorname{tag}(\mathrm{T}, \mathrm{S}, \mathrm{K}, \mathrm{Hash}, \mathrm{SND}, \mathrm{RCV})$

$\wedge$ server (S,T,K,Hash, SND, RCV)

end role

$\% \% \% \% \% \% \% \% \% \% \% \% \% \% \% \% \% \% \% \% \% \% \% \% \%$

role environment $($ ) def=

const

id, id2, id3, id4, id5,trid, trk: protocol_id,

$\mathrm{h}$ : hash_func,

k, kti,ksi: symmetric_key,

tag, server: agent

intruder_Knowledge $=\{$ tag,server,h,i,kti,ksi $\}$

composition

$$
\begin{aligned}
& \quad \text { session(tag,server,k,h) } \\
& \wedge \text { session(tag,i,kti,h) } \\
& \wedge \text { session(i,server,ksi,h) } \\
& \text { end role }
\end{aligned}
$$

$\% \% \% \% \% \% \% \% \% \% \% \% \% \% \% \% \% \% \% \% \% \% \% \% \%$ goal

secrecy_of id, id2, id3

authentication_on trid

authentication_on id3

authentication_on trk

end goal

$\% \% \% \% \% \% \% \% \% \% \% \% \% \% \% \% \% \% \% \% \% \% \% \%$ environment() 


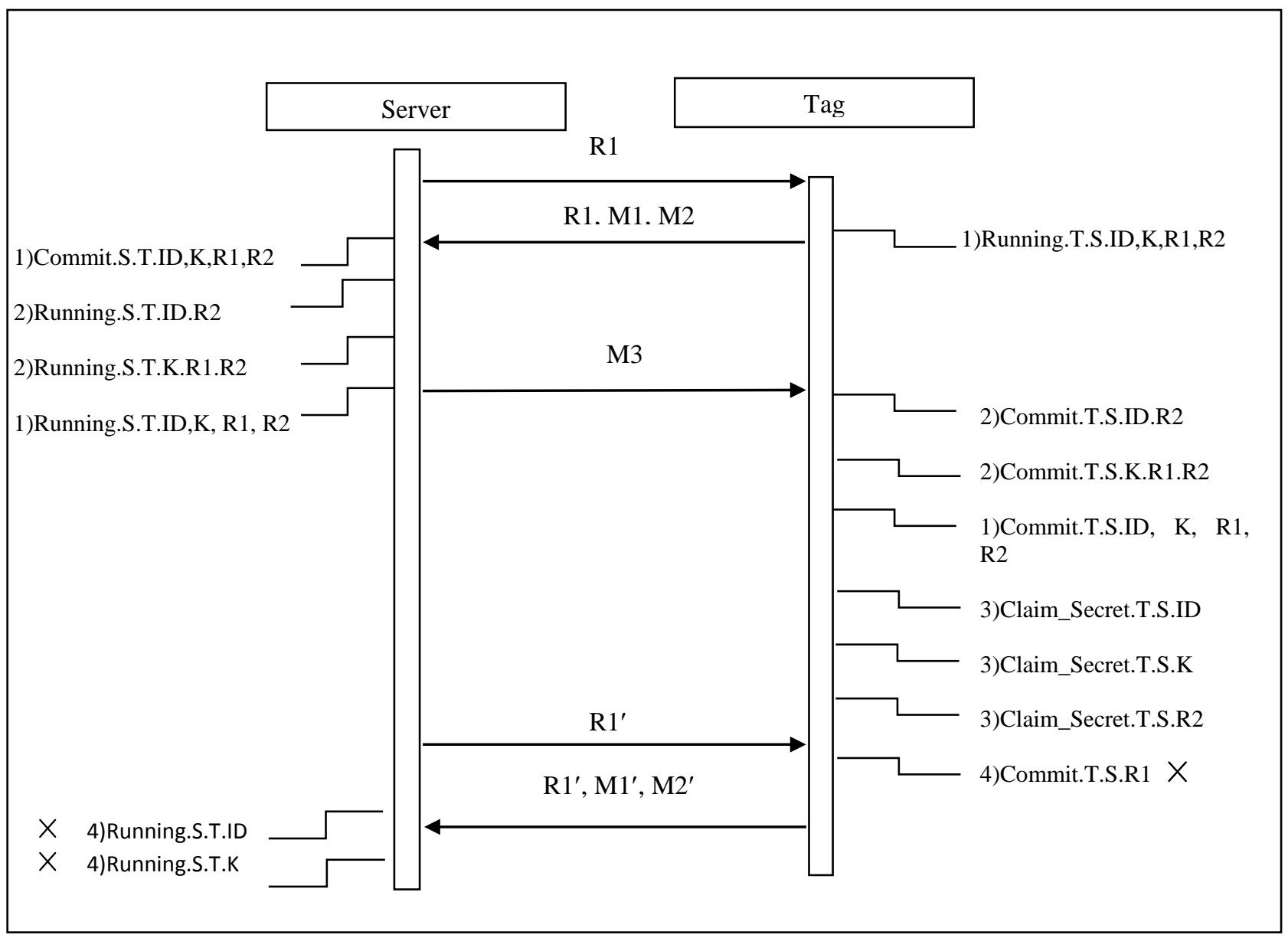

Figure1. The proposed protocol requirement illustration in CasperFDR 